\title{
Phase change materials in energy sector - applications and material requirements
}

\author{
Marta Kuta ${ }^{1, a}$ and Tadeusz M. Wójcik ${ }^{1}$ \\ ${ }^{1}$ AGH University of Science and Technology, Faculty of Energy and Fuels, Department of Thermal and Fluid Flow Machines, al. A. \\ Mickiewicza 30, 30-059 Krakow, Poland
}

\begin{abstract}
Phase change materials (PCMs) have been applying in many areas. One of them is energy field. PCMs are interesting for the energy sector because their use enables thermal stabilization and storage of large amount of heat. It is major issue for safety of electronic devices, thermal control of buildings and vehicles, solar power and many others energy domains. This paper contains preliminary results of research on solid-solid phase change materials designed for thermal stabilisation of electronic devices.
\end{abstract}

\section{Introduction}

Energy sector is important issue for the present society. It includes wide range of topics. Some of them are linked to phase change materials. Part of those applications is presented below [1].

\subsection{Building applications}

One of the most important applications of phase change materials in energy sector is use PCMs in housing and public facilities. It can be applicable in various ways, among others:

- building materials and components with the content of PCMs,

- latent heat storage containers (with PCMs) integrated in building structures.

By the use of PCMs in buildings application it is possible to reduce requirement for heat and reduce indoor overheating.

\subsection{Solar energy}

Phase change materials can be applied in solar energy application for:

- solar heating,

- solar cooling,

- solar hot water,

- photovoltaic system.

\subsection{Thermal energy storage}

Phase change materials can be applied for short-term, medium-term and long-term thermal energy storage. Selection of appropriate material depends on demand - several hours excess, daily overload of thermal energy or even seasonal heat surplus.

Thermal energy storage can have a positive impact in the form of: increasing the reliability of the system, improving the functioning of the power plants and energy systems, reducing energy purchase costs by shifting energy surplus from periods of lower to periods of higher demand.

At the time when cost of energy is getting higher and higher it is important to improve old and find new ways of energy saving. One of them is thermal energy storage and use stored energy in the future.

\subsection{Electronic devices}

There is also possibility of using PCMs for electronic devices:

- PCM battery jacket - which cover and keep battery at optimum [2],

- PCM pad - which protect portable computers from overheating [3],

- thermal management for mobile phones[4],

- heat sinks modified by use of PCMs [5].

Authors of the paper have decided to investigate one of listed above application of PCMs - for computer heat sink modification.

\section{Phase change materials}

\subsection{Phase change materials}

Phase change materials are substances characterised by ability to phase transition at certain temperature range. Scheme of the phase change transition is presented on figure 1. During phase transition there is always heat

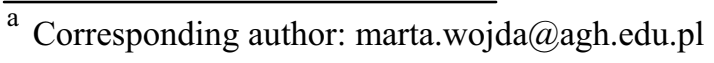


emission or heat absorption which is called latent heat of fusion. Temperature of transition and amount of latent heat of fusion are distinctive for each material. PCMs are capable to store thermal energy for short or long time.

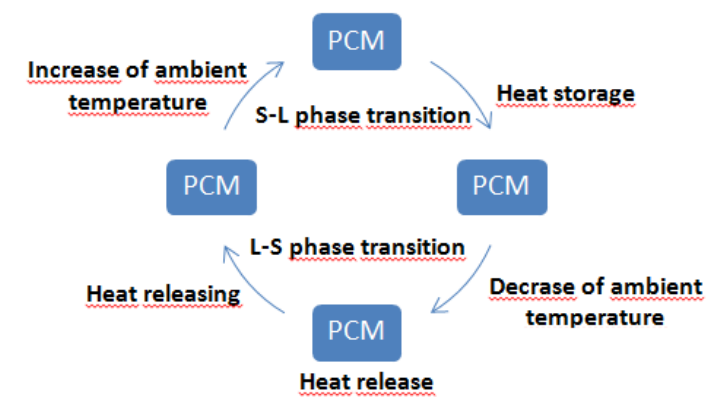

Figure 1. Solid - liquid phase change transition as an example of phase change transition cycle.

\subsection{Phase change materials classification}

There is many methods of phase change materials classification [6]. The most typical is grading with respect to type of transition:

- solid - liquid,

- solid - solid,

- gas - liquid,

- solid - gas.

Another important type of classification concern to chemical composition. It is also important because PCMs properties are strongly depend on type of composition.

Classification because of type of chemical composition is as below:

- organic,

- inorganic,

- eutectics.

\subsection{Properties of phase change materials relevant for use in the energy sector}

PCMs are selected taking into account among the othersthermophisical, structural and economical properties:

a) thermophisical:

- phase change transition temperature,

- latent heat of fusion,

- thermal conductivity,

- stability of properties at many work cycles.

b)structural:

- small volume change,

- chemical stability,

- compatibility with different materials,

- non-toxic,

- non-flammable.

c)economical:

- low price,

- recycling possibility.

The most important properties of PCMs is temperature range of phase transition. Phase change materials can be used for many applications where heat storage or temperature stabilization is needed. For example: thermal energy storage, buildings thermal stabilization, off peak power utilization, waste heat recovery, application at solar power plants, food transport, blood and medicines transport, temperature stabilization of electronic devices.

It is important to find PCM suitable for application.

\subsection{Solid - solid phase change materials}

The best known and the most commonly used are solidliquid phase change materials(S-L PCMs). However during few last years interest in solid-solid phase change materials(S-S PCMs) has grown. S-S PCMs are materials with solid-solid transition, so materials with change of crystallographic structure. The fact that typical phase transition does not occur in case of S-S PCMs favours their use for application where the following requirements are necessary to complied:

- minimal or no volume change,

- no liquid or gas generation.

Predominance of solid-solid over solid - liquid transition involves minimization of volume change or liquid/gas generation possibility. It decreases probability of corrosion or damage and enables using S-S PCMs for wide range of applications. This work contains analysis of the possibility of use PCMs for thermal stabilisation of electronic devices

\section{Experimental procedures}

\subsection{Synthesis of solid-solid phase change materials and its thermophisical properties}

Solid-solid phase change material has been synthesized in order to assess their suitability for thermal stabilisation of electronic devices. Main components of synthesized material are polyethylene glycol (PEG) and ethyl cellulose (EC). To select material characterized by suitable properties, group of materials have been synthesized, examined and their properties have been compared. Details concerning type and percentage of polyethylene glycol used for the synthesis are presented in table 1 .

Table 1. PEG type and percentage used for the synthesis

\begin{tabular}{|c|c|}
\hline PEG type & PEG percentage, $\%$ \\
\hline \multirow{3}{*}{ PEG 2000 } & 50 \\
\cline { 2 - 2 } & 65 \\
\cline { 2 - 2 } & 75 \\
\cline { 2 - 2 } & 80 \\
\hline \multirow{4}{*}{ PEG 4000 } & 50 \\
\cline { 2 - 2 } & 65 \\
\cline { 2 - 2 } & 75 \\
\hline \multirow{4}{*}{ PEG 8000 } & 80 \\
\cline { 2 - 2 } & 50 \\
\cline { 2 - 2 } & 65 \\
\cline { 2 - 2 } & 75 \\
\hline \multirow{4}{*}{12000} & 80 \\
\cline { 2 - 2 } & 50 \\
\cline { 2 - 2 } & 65 \\
\cline { 2 - 2 } & 75 \\
\hline
\end{tabular}


It has been audited that increase of content of polyethylene glycol in the synthesized material affects the growth of latent heat of fusion of this material. Also type of used polyethylene glycol is important-the higher molecular weight of PEG - the higher value of latent heat of fusion.

Simultaneously, it has been observed that if it is used too little cellulose, it is not possible to obtain solid-solid phase change material. This is because the cellulose is responsible for the creation of a rigid structure which is filled with polyethylene glycol. PEG aimed to give characteristics typical for phase change materials.

Figure 2 present results of Fourier transform infrared spectroscopy for synthesized material (PEG 4000-75\%) and used for its synthesis: pure PEG 4000 and cellulose. It can be inferred that polyethylene glycol has been grafted into the cellulose frame.

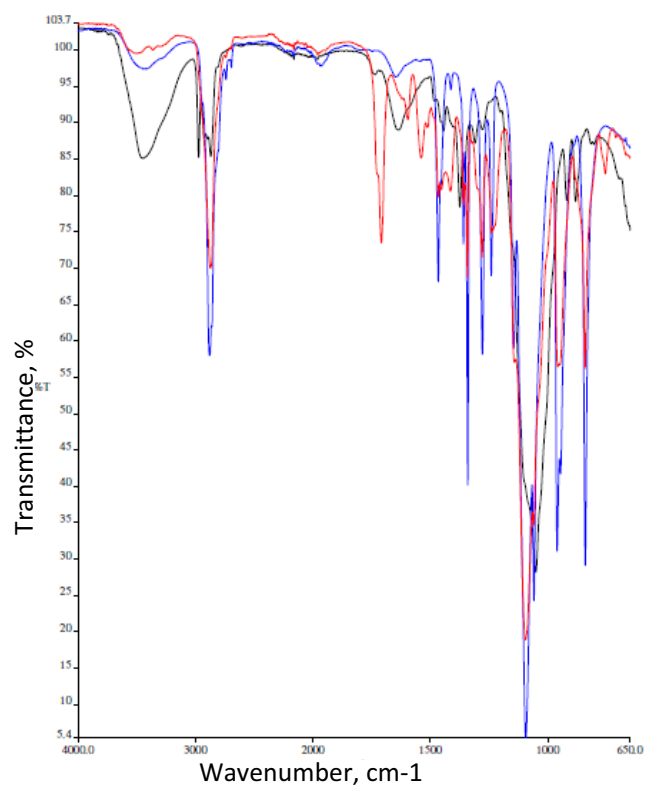

Figure 2. FTiR results for cellulose (black), pure PEG 4000 (blue) and S-S PCM containing 75\% of PEG4000.

Mentioned dependencies were observed with use of differential scanning calorimetry (DSC) technic. Two charts below - figure 3 and 4 present relations for material synthesized with use of different PEG type(Figure 3 ) and with use of various mass ratio of PEG4000 (Figure 4).

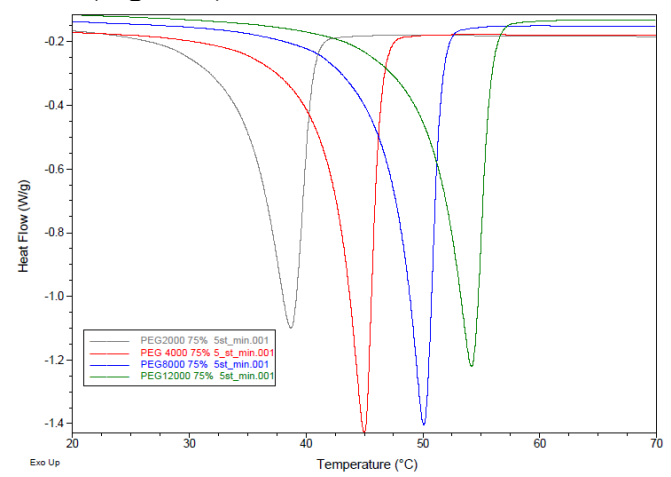

Figure 3. DSC results- curves for materials with different type of polyethylene glycols: 2000(grey), 4000 (red), 8000 (blue), 12000 (green).

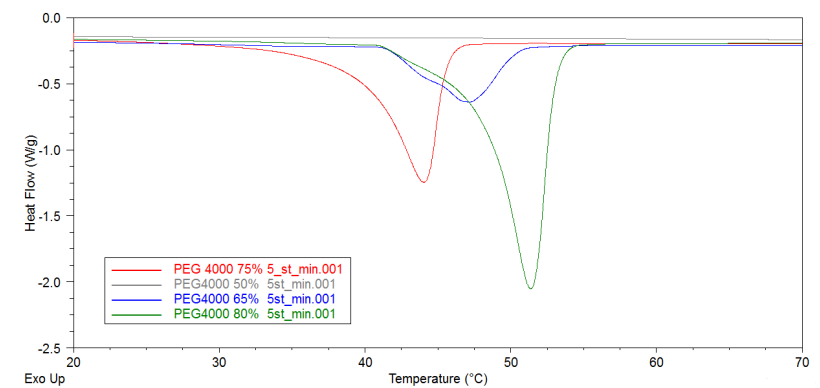

Figure 5. DSC results- curves for materials with different PEG4000 ratio: 50\%(grey), 65\% (blue), 75\% (red), 80\%(green).

Material has been synthesized taking into account several important requirements:

- Properly selected transition temperature. Temperature in the range of $40-50^{\circ} \mathrm{C}$ has been chosen considering the maximum temperature of safe work for selected computer processors.

- As large as possible value of latent heat of fusion. Due to quite high value of latent heat of fusion and suitable transition temperature material containing $75 \%$ of $\mathrm{PEG}$ 4000 has been chosen. Heat of fusion and transition temperature of synthesized material are presented in table 2 .

Table 2. Heat of fusion and transition temperature for synthesized material (PCM containing $75 \%$ of PEG4000)

\begin{tabular}{|c|c|c|}
\hline \multicolumn{2}{|c|}{ Phase change temperature, ${ }^{\mathbf{0}} \mathbf{C}$} & \multirow{2}{*}{ Heat of fusion, $\mathbf{J} / \mathbf{g}$} \\
\hline Onset & 41,0 & \multirow{2}{*}{61,4} \\
\hline Endset & 48,5 & \\
\hline Peak & 44,5 & \\
\hline
\end{tabular}

-As large as possible value of thermal conductivity Due to improve thermal conductivity selected material has been modified with use of graphite and carbon nanotubes. Percentages of additives used for synthesis are presented in table 3 .

Table 3. Type and percentage of appendix

\begin{tabular}{|c|c|}
\hline \multirow{2}{*}{ Appendix type } & $\begin{array}{c}\text { Appendix } \\
\text { percentage, } \\
\%\end{array}$ \\
\hline \multirow{2}{*}{ Graphite } & 5 \\
\cline { 2 - 2 } & 10 \\
\cline { 2 - 2 } & 25 \\
\hline \multirow{2}{*}{$\begin{array}{c}\text { Carbon } \\
\text { nanotubes }\end{array}$} & 5 \\
\cline { 2 - 2 } & 10 \\
\cline { 2 - 2 } & 25 \\
\hline
\end{tabular}

The best solution will be selected taking into consideration degree of thermal conductivity and economic reasons.

Figure 6 presents Scanning Electron Microscope (SEM) image of PCM PEG4000-75\% and its composite with $10 \%$ of multiwall carbon nanotubes. It can be observed that cellulose and PEG in synthesized material are well consistent and carbon nanotubes are dispersed uniformly. It suggest that components of PCM are compatible and carbon nanotubes distribution is quasi uniform. It can 
indicate that thermal conductivity of PCM will be significantly improved.

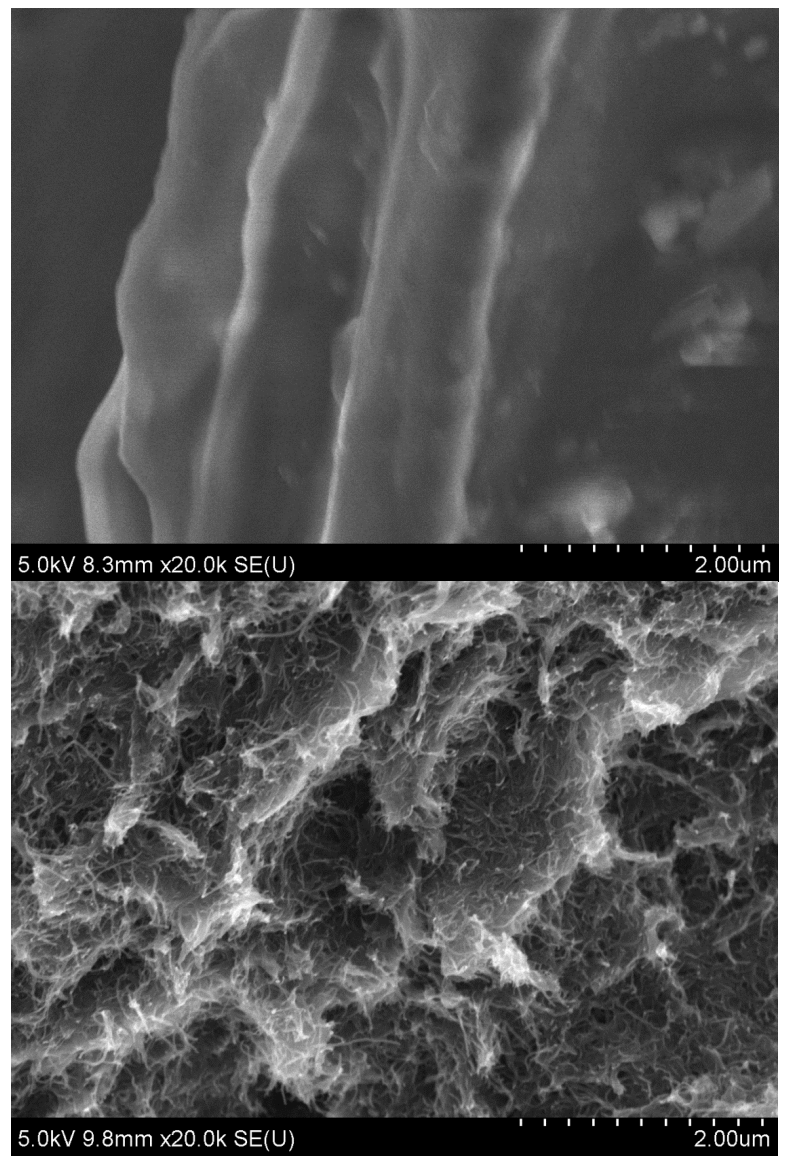

Figure 6. SEM of ( a) PCM PEG4000-75\% (b)composite: PCM PEG4000-75\% with carbon nanotubes(10\%).

-Structural properties: small volume change, no leakage problem, chemical stability, compatibility with different materials, non- toxic.

All those requirements have been included at the project stage. Solid-solid PCM has been chosen to avoid changes in the volume and the problem of leakage.

- Stability of properties at many work cycles

To assess the ability to work in multiple cycles material will be tested on the testing rig.

\subsection{Testing rig}

Synthesized phase change material will be tested in order to:

- examine stability of material properties after working in multiple cycles,

- compare work characteristic for heating and cooling cycles,

- determination of characteristics of enthalpy with temperature,

- determination of characteristics of specific heat with temperature.

Tests will be carried out on the testing rig consisting of: main module, heating system with power measurement, computer, temperature measurement system, image acquisition system, data acquisition system.

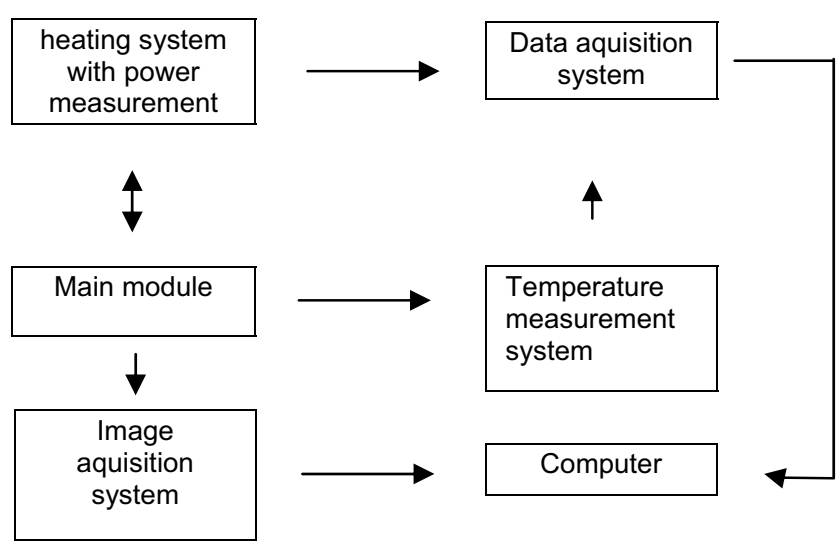

Figure 7. Scheme of the testing rig.

\section{Conclusions}

Energy sector is growing and it is important to find new and improve the existing technologies. One of the possibilities which can be used to achieve this goal is application of phase change materials. Due to great advantages solid-solid PCMs are becoming more and more popular.

Group of novel solid-solid phase change materials based on polyethylene glycol and cellulose has been synthesized. Materials were examined and one of them has been chosen for further work. Chosen material will be tested for the possibility of thermal stabilisation of electronic devices. Preliminary results of tests on the material confirmed possibility of its use for mentioned application.

\section{Acknowledgments}

This work was supported by AGH - University of Science and Technology (Project 11.11.210.216).

\section{References}

1. T.M. Wójcik, R.Pastuszko, M.Wojda, W. Kalawa, EPJ Web of Conferences 67, 02130 (2014).

2. International Energy Agency Implementing Agreement on Energy Conservation through Energy Storage, Annex 17, "Advanced Thermal Energy Storage through Phase Change Materials and Chemical Reactions - Feasibility Studies and Demonstration projects", Final Report

3. W. Xiong, Patent: Cooling pad for laptop computer

4. A.P. Cosentino, Telecommunications Energy Conference, 2000. INTELEC, 237 - 244 (2000)

5. M.Wojda, T.M.Wójcik, The MATEC Web of Conferences (to be published)

6. B. Zalba, J. M. Marin, L.F.Cabeza, H. Mehling, Applied Thermal Engineering 23, 251 (2003) 